\title{
Acute consumption of fish oil improves postprandial VLDL profiles in healthy men aged 50-65 years
}

\author{
Graham C. Burdge ${ }^{1}$, Jonathan Powell ${ }^{2}$, Tony Dadd ${ }^{2}$, Duncan Talbot ${ }^{2}$, Jacqueline Civil ${ }^{2}$ \\ and Philip C. Calder ${ }^{1}$ \\ ${ }^{1}$ Institute of Human Nutrition, Southampton General Hospital, University of Southampton, Developmental Sciences Building, \\ Mail point 887, Tremona Road, Southampton SO16 6YD, UK \\ ${ }^{2}$ Unilever R\&D Colworth, Colworth Science Park, Sharnbrook, Bedford MK44 1LQ, UK
}

(Received 28 July 2008 - Revised 2 October 2008 - Accepted 22 October 2008 - First published online 13 January 2009)

Dietary supplementation with fish oil induces beneficial changes in the size and concentration of plasma lipoproteins, although the underlying mechanism is unclear. We have investigated the effect of increasing the amount of fish oil in a single meal on the size and concentration of VLDL, LDL and HDL particles during the postprandial period. Healthy men aged 58 (SD 5) years $(n$ 11) consumed isoenergetic, mixed macronutrient test meals containing either $0.3 \mathrm{~g}$ (reference, REF) or $2 \cdot 2 \mathrm{~g}$ (high fish oil, HFO) fish oil in a randomised order, and blood samples were collected over the following $6 \mathrm{~h}$. Plasma lipoprotein size and concentration were measured by NMR spectroscopy. There was a significant interaction effect of time and meal composition on the VLDL, but not on the LDL or HDL, concentration $(P=0 \cdot 036)$ and particle size $(P=0 \cdot 005)$. Consuming the HFO meal significantly increased the VLDL concentration $(P<0.05)$ and reduced VLDL particle size $(P<0.05)$ when compared with the REF meal and baseline. LDL particle size decreased slightly during the postprandial period, but there was no difference between the meals. There was no effect of time or meal composition in the LDL concentration. The HDL concentration decreased and size increased slightly during the postprandial period, but there were no significant differences between the meals. Increased consumption of fish oil induces acute changes in the VLDL, but not in the LDL or HDL, metabolism.

Lipoprotein: Postprandial: Fish oil

Increased risk of CVD is associated with specific changes in the size and number of individual lipoprotein particles. For example, low HDL-cholesterol and high LDL-cholesterol are independent risk factors for $\mathrm{CVD}^{(1-3)}$. The fasting and postprandial TAG concentrations have also been identified as risk factors and are involved in a causal manner in the pathogenesis of $\mathrm{CVD}^{(4,5)}$. At least, part of the link between the plasma TAG concentration and the CVD risk lies in the relationship between the size of VLDL and LDL particles, and the extent of the exchange of TAG and cholesteryl esters between VLDL and HDL particles. LDL particles are generated from VLDL remnants. Larger, TAG-rich VLDL $_{1}$ particles give rise to a range of LDL particles including small, dense $\mathrm{LDL}_{3}$, which have been suggested to be atherogenic $^{(3,6)}$, although not all studies support this view ${ }^{(7,8)}$. Smaller $\mathrm{VLDL}_{2}$ particles are the precursors of mainly $\mathrm{LDL}_{1}$ and $\mathrm{LDL}_{2}{ }^{(3,6,9)}$. The total $\mathrm{LDL}$ particle concentration may also contribute to the risk of $\mathrm{CVD}^{(10)}$. Higher VLDL-TAG concentrations are associated with increased exchange of TAG, with HDL-cholesteryl esters leading to the removal of HDL from the circulation and enrichment of VLDL remnants with cholesteryl esters, which contributes to the generation of atherogenic $\operatorname{LDL}^{(3,6,9)}$.
An increased intake of fish oil containing $n$ - 3 fatty acids is associated with a lower risk of $\mathrm{CVD}^{(11-13)}$. A review of seventy-two studies that investigated the effect of increasing dietary intake of fish oil rich in very-long-chain $n$-3 PUFA, DHA $(22: 6 n-3)$ and EPA $(20: 5 n-3)$ on plasma lipoprotein profiles concluded that supplementation with fish oil lowered fasting plasma TAG and increased the LDL-cholesterol concentration, while the HDL-cholesterol concentration was unaffected ${ }^{(14)}$. Overall, dietary supplementation with $n-3$ PUFA at approximately $4 \mathrm{~g} / \mathrm{d}$ induces a decrease in the fasting plasma TAG concentration of $25-30 \%{ }^{(14)}$. Although the reduction in the plasma TAG concentration may be beneficial, the increase in LDL-cholesterol is potentially harmful if it reflects an increase in the number of small atherogenic LDL particles $^{(15,16)}$. EPA and DHA given as individual fatty acids tend to reduce the fasting TAG concentration to a similar extent ${ }^{(17,18)}$, while reports differ in the extent and direction of the effects of these fatty acids on the LDL- and HDLcholesterol concentrations ${ }^{(19)}$.

The precise mechanism by which fish oil induces the lower plasma TAG concentration is a matter of debate ${ }^{(20)}$, but involves changing the size distribution and concentration of plasma lipoproteins ${ }^{(19,21,22)}$. Many of the effects of EPA and 
DHA on the cell function are dependent upon the incorporation of these fatty acids into membrane phospholipids over a prolonged period of time ${ }^{(23)}$. However, the TAG lowering effect of fish oil also involves the direct effects of EPA or DHA, or their peroxidation products, on specific transcription factors in the liver ${ }^{(20)}$. The overall effect of activation of these transcription factors is to modify the metabolic partitioning of fatty acids away from storage towards $\beta$-oxidation, thus reducing the availability of TAG for incorporation into TAG and secretion into the circulation. Administration of a hyperinsulinaemic-euglycaemic clamp reduced VLDL secretion, possibly by reducing the flux of NEFA to the liver ${ }^{(24)}$. Thus, VLDL-TAG secretion may be regulated by the changes in the supply of fatty acid substrates to the liver. DHA was incorporated preferentially into the NEFA pool within 90 min following meals containing 2 or $30 \mathrm{~g}$ fish oil ${ }^{(21,22,25,26)}$ and incorporated into VLDL-TAG within $3 \mathrm{~h}$ when compared with $\mathrm{EPA}^{(25)}$. This suggests that DHA released from chylomicron TAG by the lipoprotein lipase activity is relatively poorly trapped, and is rapidly assimilated by the liver and incorporated into TAG destined for secretion in $\mathrm{VLDL}^{(25)}$. This raises the possibility that an acute increase in fish oil intake may induce rapid changes in the hepatic VLDL metabolism. If so, then the differences between individuals in the regulation of fatty acid partitioning in the liver may contribute to any variation in the effectiveness of fish oil supplementation in reducing the plasma TAG concentration and hence the risk of CVD. Fish oil may also alter the postprandial TAG metabolism by changing the competition between chylomicrons and VLDL for lipoprotein lipase, and hence altering the rate of hydrolysis and/or altering the interactions between circulating lipoproteins such as the exchange of apolipoproteins.

The aim of the present study was to test the hypothesis that increasing the fish oil content of a single meal alters the size and number of VLDL, LDL and HDL particles in the plasma during the postprandial period. Healthy men aged 50-65 years consumed either a REF meal or a meal containing 7.3-fold more EPA and DHA in a random order, and lipoprotein size and number was measured over the subsequent $6 \mathrm{~h}$ period by NMR spectroscopy.

\section{Materials and methods}

\section{Subjects}

Details of the subjects and the postprandial study have been reported previously ${ }^{(27)}$. The subjects were eleven healthy men aged 58 (SD 5) years, who had a BMI of $25 \cdot 8$ (SD 3.3) $\mathrm{kg} / \mathrm{m}^{2}$, a fasting TAG concentration of $1.3(\mathrm{SD} 0.4) \mathrm{mmol} / \mathrm{l}$, a fasting total cholesterol concentration of 4.7 (SD 0.7$) \mathrm{mmol} / \mathrm{l}$, a fasting glucose concentration of 5.0 (SD 0.4 ) $\mathrm{mmol} / \mathrm{l}$ and a fasting insulin concentration of 4.6 (SD 2.6; mU/l) measured at a pre-study health screen. The volunteers who consumed restricted diets (e.g. vegetarians and vegans), consumed more than two portions of oily fish per week, habitually took vegetable or fish oil or vitamin dietary supplements, had diagnosed type 2 diabetes mellitus or smoked tobacco were excluded from the study. The study received ethical approval from the South and West Hampshire Local Research Ethics Committee. All volunteers gave their written informed consent.

\section{Study design}

Each participant took part in the two postprandial studies in a randomised order, with an interval of at least $14 \mathrm{~d}$ between each study ${ }^{(27)}$. The subjects consumed their habitual diet throughout the study. On the day preceding a postprandial study, the subjects were asked not to consume alcohol and to refrain from strenuous exercise. They were asked to consume their evening meal by 20.00 hours, but could consume water freely, and to fast until the postprandial study commenced. The subjects arrived at the Wellcome Trust Clinical Research Facility (Southampton General Hospital, Southampton, UK) at approximately 07.30 hours. A cannula was inserted into a forearm vein and a baseline blood sample $(12 \mathrm{ml})$ drawn. Part of the sample $(10 \mathrm{ml})$ was placed into a tube containing heparin sulphate, and the remainder was placed into a tube and allowed to clot. The participants were given one of two test meals at approximately 08.00 hours. These were a reference (REF) meal based on the pattern of fatty acids in the UK diet or a meal with the increased EPA and DHA content (high fish oil, HFO) ${ }^{(27)}$. The macronutrient and fatty acid compositions of the test meals have been reported in detail previously ${ }^{(27)}$ and are summarised in Table 1. The fat and protein component of the meals was administered in an emulsion ${ }^{(27)}$ that was composed of double cream, a blend of sunflower, olive, linseed and fish oils, Nesquik milkshake powder, casein and carbohydrate. The total volume was made up to $160 \mathrm{ml}$ with water. The carbohydrate component of the meal was given as toast with jam or marmalade. The subjects consumed the test meal during a period of approximately $15 \mathrm{~min}$. The blood samples $(12 \mathrm{ml})$ were collected at $0.5,1,1.5,2,2.5,3,4,5$ and $6 \mathrm{~h}$ after the consumption of the test meal began. The participants remained resting throughout the study and were allowed free access to water.

Measurements of lipoprotein particle size and number, and of 8-isoprostane

The plasma was separated from the cells by centrifugation and frozen. The number and size of particles within VLDL, LDL

Table 1. Composition of the test meals

\begin{tabular}{lrr}
\hline & REF meal & HFO meal \\
\hline Total fat $(\mathrm{g})$ & $55 \cdot 1$ & $55 \cdot 9$ \\
Total carbohydrate $(\mathrm{g})$ & $130 \cdot 0$ & $130 \cdot 0$ \\
Total protein $(\mathrm{g})$ & $12 \cdot 0$ & $12 \cdot 0$ \\
Total energy (MJ) & $4 \cdot 3$ & $4 \cdot 3$ \\
Fatty acid composition $(\mathrm{g})$ & & \\
$12: 0$ & $1 \cdot 0$ & $0 \cdot 9$ \\
$14: 0$ & $3 \cdot 5$ & $3 \cdot 5$ \\
$16: 0$ & $11 \cdot 8$ & $11 \cdot 5$ \\
$18: 0$ & $4 \cdot 6$ & $4 \cdot 2$ \\
$16: 1 n-7$ & $0 \cdot 8$ & $1 \cdot 4$ \\
$18: 1 n-9$ & $18 \cdot 6$ & $17 \cdot 4$ \\
$18: 2 n-6$ & $12 \cdot 1$ & $10 \cdot 6$ \\
$18: 3 n-3$ & $2 \cdot 0$ & $1 \cdot 8$ \\
EPA & $0 \cdot 2$ & $1 \cdot 1$ \\
$22: 5 n-3$ & $0 \cdot 1$ & $0 \cdot 2$ \\
DHA & $0 \cdot 2$ & $1 \cdot 1$ \\
Total EPA + DHA & $0 \cdot 3$ & $2 \cdot 2$ \\
\hline
\end{tabular}

REF, reference; HFO, high fish oil. 
and HDL lipoprotein classes were measured by NMR spectroscopy (LipoScience, Inc., Raleigh, NC, USA). This method has been validated in the studies of lipoproteins in a number of physiological and pathological states ${ }^{(28)}$, which include the analysis of samples from fasting ${ }^{(29-32)}$ and postprandial $^{(33)}$ subjects. The particle concentrations were determined from the amplitudes of their spectroscopically distinct NMR signals and weighted average particle diameters were derived from the sum of the diameters of each lipoprotein class multiplied by its relative mass ${ }^{(19,34,35)}$. The analyses were performed in accordance with commercial quality control measures. To determine whether fish oil consumption increased the level of lipid peroxides, the plasma 8-isoprostane concentration was measured by DELFIA (Perkin-Elmer, Waltham, MA, USA) time-resolved competitive fluorescence immunoassay using a monoclonal antibody, which has been described previously ${ }^{(36)}$.

\section{Statistical analysis}

The data were analysed using a standard linear mixed-modelling approach with repeated measures in Statistical Analysis Systems statistical software package version 9.1.3 via Proc Mixed (SAS Institute, Cary, NC, USA), the response variable being the change from baseline. Fixed effects were included in the model to account for the baseline value within the subject/diet combination according to standard practice $^{(37)}$, and the diet $\times$ time interaction. Random effects were also included to account for subject and subject $x$ diet interaction. The diet $\times$ time interaction term was then decomposed in order to provide an estimate for the difference between diets at each time point. No correction for multiple testing was made. The studentised conditional residuals from each model were inspected in order to assess the model fit and identify the presence of outliers.

\section{Results}

All subjects completed both postprandial studies. All data are presented as means with their standard errors for a total of eleven subjects. There were significant differences between the meals at baseline for VLDL particle size (REF 52.1 (SD 2.9) nm, HFO 48.4 (SD 2.8) nm; paired Student's $t$ test, $P=0.021)$. There were no significant differences between the meals at baseline for VLDL concentration (REF 72.9 (SD 10.8) nmol/l, HFO 66.2 (SD 30.1) nmol/l), LDL particle size (REF 21.1 (SD 0.2) nm, HFO 21.2 (SD 0.3) nm) or concentration (REF 1148.8 (SD 131.3) nmol/l, HFO 1141.5 (SD 134.4) nmol/l), or HDL particle size (REF 8.8 (SD 0.1) nm, HFO 8.8 $(\mathrm{SD} 0 \cdot 1) \mathrm{nm}$ ) or concentration (REF $31 \cdot 1$ (SD 1.2) $\mu \mathrm{mol} / \mathrm{l}$, HFO $30 \cdot 4(\mathrm{sD} 1 \cdot 3) \mu \mathrm{mol} / \mathrm{l})$.

There was a significant interaction effect of meal composition and time $(P=0.036)$ on the VLDL concentration (Fig. 1). There was no significant change in the concentration of VLDL particles when compared with baseline following the REF meal. However, following the HFO meal, the concentration of VLDL particles was significantly increased when compared with baseline between 3 and $6 \mathrm{~h}$. Between 3 and $5 \mathrm{~h}$, the change in the VLDL concentration from baseline was significantly greater following the HFO meal when compared with the REF meal. There was a significant interaction effect of meal composition and time $(P=0.005)$ on VLDL particle size. Consuming the REF meal increased the size of VLDL particles when compared with baseline between 1 and $6 \mathrm{~h}$. Consuming the HFO meal also significantly increased the size of VLDL particles between 1 and $4 \mathrm{~h}$, but returned to a level not significantly different from baseline by $5 \mathrm{~h}$. Between 2.5 and $4 \mathrm{~h}$, VLDL particles were significantly smaller $(P<0.05$ to $P<0.001)$ after the HFO meal than the REF meal.

There was no significant effect of time or meal composition on the LDL concentration (Fig. 1). There was a significant effect of time $(P=0 \cdot 013)$, but not meal composition, on LDL particle size. Following both the meals, LDL particle size was significantly reduced when compared with baseline between 2 and $5 \mathrm{~h}$.

There was a significant effect of time $(P=0 \cdot 0002)$, but not meal composition, on the HDL concentration. The HDL concentration was significantly lower than baseline between 2.5 and $4 \mathrm{~h}$ following the REF meal, and between 3 and $4 \mathrm{~h}$ following the HFO meal (Fig. 1). There was also a significant effect of time $(P<0.0001)$, but not meal composition, on HDL particle size. There was an increase in the HDL size following both the meals, but this was only significant following the HFO meal.

There was no significant effect of diet or time on the 8-isoprostane concentration (data not shown).

\section{Discussion}

The results of the present study show, for the first time, that increasing the amount of fish oil in a single meal induces class-specific changes in the concentration and particle size of lipoproteins. Consuming the HFO meal increased the VLDL concentration and decreased the VLDL size during the postprandial period. An acute increase in the consumption of fish oil did not significantly alter the LDL or HDL concentration or the particle size. There were modest, but highly variable, changes in the LDL size and in the HDL concentration and size in the late postprandial period.

A number of studies have reported that increased consumption of fish oil, or purified EPA or DHA, lowers the fasting or postprandial TAG concentration ${ }^{(14)}$, and that these fatty acids change the particle size distribution and concentration of specific lipoproteins ${ }^{(19)}$. Since these studies involve increased intake of EPA and/or DHA over a prolonged period, the findings imply the induction of metabolic adaptation rather than an acute effect. The mechanism through which $n-3$ PUFA lower the plasma TAG concentration is unclear, although it has been argued that altered metabolic partitioning of the fatty acids is critical to this process ${ }^{(20)}$. In the present study, we present data which show that consuming a single meal enriched with fish oil significantly alters both the VLDL concentration and particle size, and that this effect is specific to VLDL particles. We have shown in the same samples that consuming the HFO meal did not alter the magnitude of the change in total plasma $\mathrm{TAG}^{(27)}$. It is possible that any effect of the HFO meal on VLDL-TAG may have been masked by chylomicron TAG from the meal. However, the present data suggest that although the total plasma TAG concentration was not altered by increasing fish oil intake, there was a shift in the VLDL metabolism towards the secretion of a greater number of smaller VLDL particles. These changes are in 
(a)

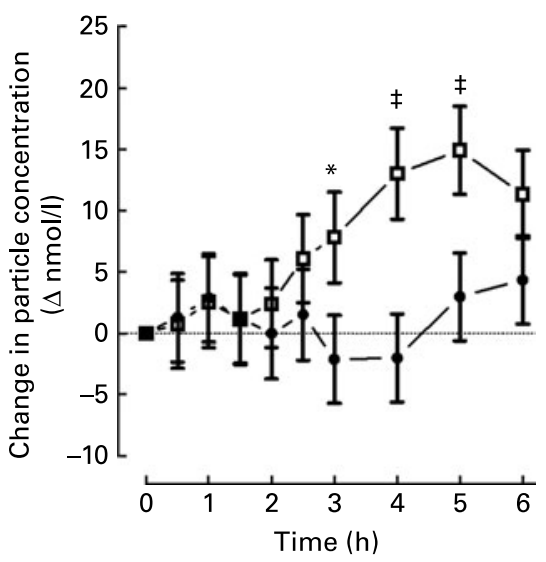

REF

HFO

(b)

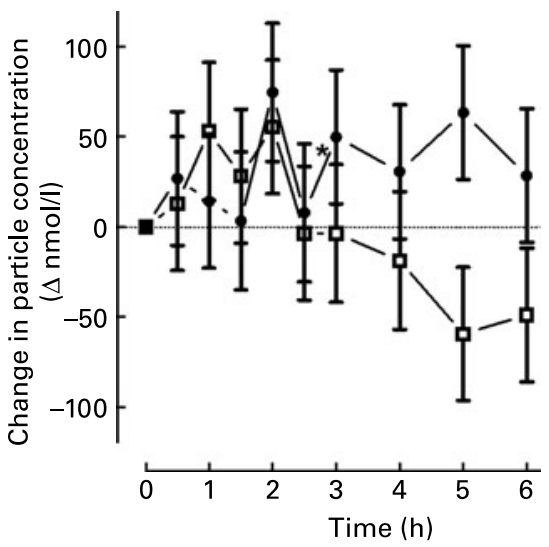

REF

HFO

(c)

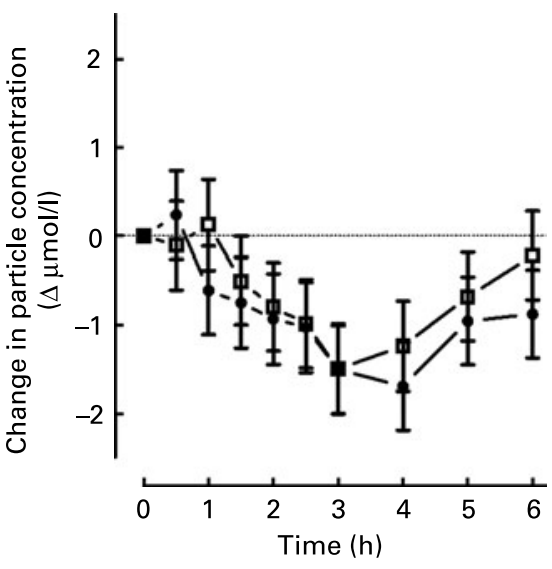

REF

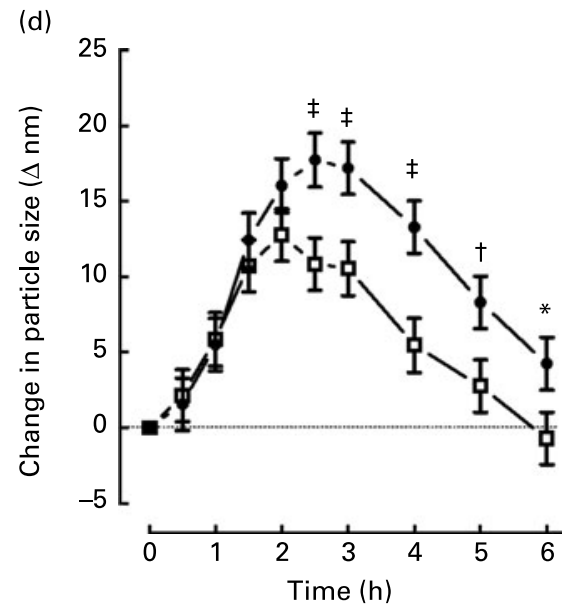

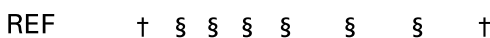

$\mathrm{HFO}+\mathrm{H}_{\mathrm{N}} \S \S \S+$

(e)

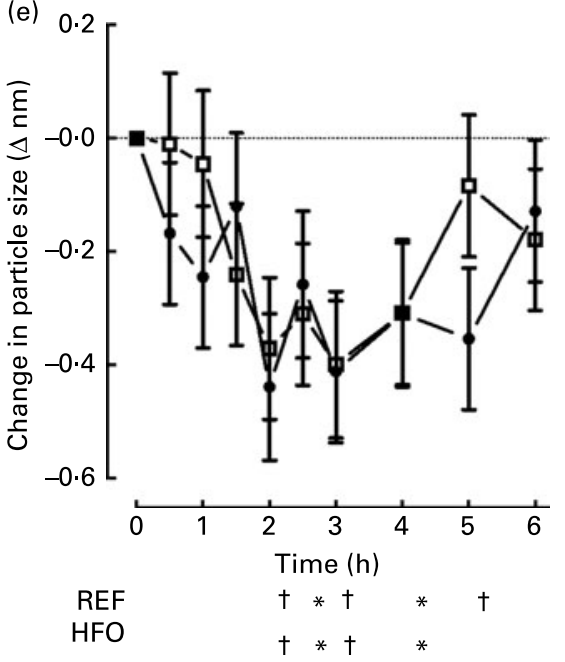

(f)

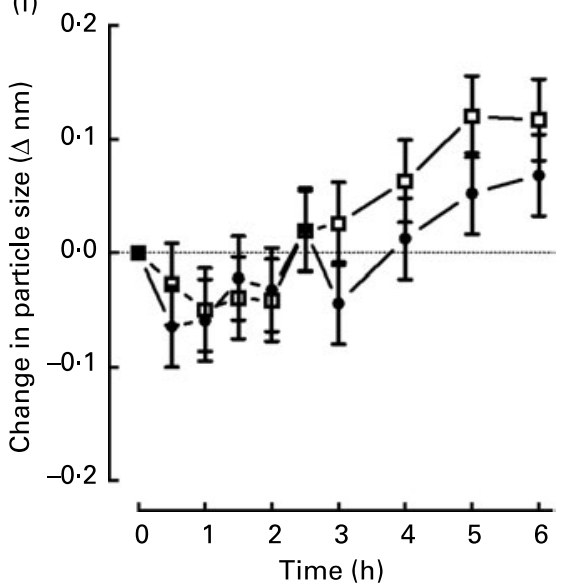

REF

$\mathrm{HFO}$

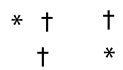

HFO

Fig. 1. Postprandial lipoprotein concentration ((a) VLDL, (b) LDL and (c) HDL) and size ((d) VLDL, (e) LDL and (f) HDL) corrected for baseline values (means with their standard errors) following the consumption of the reference ( $\square-$; REF) or high fish oil ( - - ; HFO) meals in men ( $n$ 11). Data were analysed using a standard linear mixed-modelling approach with time and meal composition as the fixed factors. The different symbols indicate different levels of statistical significance: ${ }^{\star} P<0.05, \dagger P<0.01, \ddagger P<0.001$ and $\S P<0.0001$. The symbols on the graph indicate the differences between the REF and HFO meals at individual time points. The symbols below the $x$-axis indicate statistically significant differences from baseline following each meal. 
agreement with the effects of dietary supplementation with fish oil on the VLDL size and concentration ${ }^{(19)}$. Thus, the metabolic basis of the beneficial effects of increased fish oil intake on the plasma TAG concentration may reflect acute changes in the regulation of VLDL particle size and secretion. Smaller VLDL particles are associated with the generation of larger LDL particles and thus lower atherogenic risk ${ }^{(1)}$. Thus, these findings, with respect to VLDL, are in agreement with the beneficial effects of fish oil on CVD risk $^{(17)}$.

There were small postprandial changes in LDL and HDL particles, but these were not affected by the amount of fish oil in the meal. The postprandial changes in the LDL metabolism have been reported previously ${ }^{(6)}$. It is possible that significant changes in the LDL or HDL metabolism associated with fish oil supplementation ${ }^{(18)}$ may have occurred after the period of blood sampling and so were not detected, or that repeated exposure to increased fish oil intake is required to modify the LDL or HDL size and concentration substantially.

Given that significant enrichment of EPA and DHA into cell membranes in human subjects due to increased dietary intake of these fatty acids occurs over a period of several weeks or more ${ }^{(23)}$, the present findings do not support altered membrane fatty acid composition as the primary mechanism underlying the effect of fish oil on the VLDL metabolism. One explanation for the effect of fish oil on the VLDL metabolism is an acute change in the activity and/or expression of transcription factors that are regulated by the fatty acids, including HNF4a, PPAR $\alpha$, PPAR $\gamma$ and SREBP1c, and that modify the fatty acid metabolism ${ }^{(20)}$. We have shown previously that DHA released from chylomicron TAG is preferentially incorporated into the plasma NEFA pool ${ }^{(26)}$ and incorporated into VLDL-TAG within the postprandial period ${ }^{(25)}$. This supports the suggestion that the fatty acids released from fish oil reach the liver within the postprandial period, and hence may be able to interact with transcription factors that regulate the hepatic fatty acid metabolism. It has been suggested that fatty acid oxidation products are more potent agonists of the transcription factors that modify metabolic partitioning of the fatty acids in the liver ${ }^{(20)}$. However, there was no indication of increased lipid peroxidation following the HFO meal when compared with the REF meal, which suggests that any direct effect of the fatty acids on the regulation of the VLDL metabolism was not mediated primarily through lipid peroxides. The reduction in the NEFA flux to the liver has been suggested to explain the reduction in the VLDLTAG concentration during a hyperinsulinaemic-euglycaemic clamp $^{(24)}$. However, we have shown in the same samples as those used for lipoprotein measurements that there was no effect of the HFO meal when compared with the REF meal on the plasma NEFA concentration during the postprandial period $^{(26)}$. This supports the suggestion that the effects of the HFO meal on the VLDL metabolism were not due to the lower flux of fatty acids to the liver. However, in the absence of measurements of chylomicron size and concentration, it is also possible that the acute effects of fish oil on the VLDL metabolism may also be due to a change in the competition between VLDL and chylomicrons for lipoprotein lipase, and hence their relative rates of hydrolysis.

Irrespective of the underlying mechanism, these findings suggest that the beneficial effects of fish oil on circulating VLDL levels reflect the acute changes in the VLDL metabolism. Thus, one possible implication is that the variation between individuals in the extent to which fish oil lowers the fasting or postprandial TAG concentration may reflect the differences in the function of pathways that regulate the VLDL metabolism.

\section{Acknowledgements}

We thank the study participants and the staff of the Wellcome Trust Clinical Research Facility, Southampton General Hospital, UK for their time and commitment. We also thank LipoScience for carrying out the NMR analysis. G. C. B., J. P., D. T. and P. C. C. designed the study. G. C. B. carried out the study, and G. C. B. and D. T. carried out the analysis. T. D. and J. C. carried out the statistical analysis. G. C. B. wrote the manuscript with contributions from P. C. C., J. P., T. D. and D. T. None of the authors had any financial or personal conflicts of interest. The study was funded by a grant from Unilever R\&D Colworth to G. C. B. and P. C. C.

\section{References}

1. Schaefer EJ (2002) Lipoproteins, nutrition, and heart disease. Am J Clin Nutr 75, 191-212.

2. Kris-Etherton PM, Harris WS \& Appel LJ (2002) Fish consumption, fish oil, omega-3 fatty acids, and cardiovascular disease. Circulation 106, 2747-2757.

3. Griffin BA (1999) Lipoprotein atherogenicity: an overview of current mechanisms. Proc Nutr Soc 58, 163-169.

4. Patsch JR, Miesenbock G, Hopferwieser T, et al. (1992) Relation of triglyceride metabolism and coronary artery disease. Studies in the postprandial state. Arterioscler Thromb 12, 1336-1345.

5. Roche HM \& Gibney MJ (1995) Postprandial triacylglycerolaemia-nutritional implications. Prog Lipid Res 34, 249-266.

6. Blackburn P, Cote M, Lamarche B, et al. (2003) Impact of postprandial variation in triglyceridemia on low-density lipoprotein particle size. Metabolism 52, 1379-1386.

7. El Harchaoui K, van der Steeg WA, Stroes ES, et al. (2007) Value of low-density lipoprotein particle number and size as predictors of coronary artery disease in apparently healthy men and women: the EPIC-Norfolk Prospective Population Study. J Am Coll Cardiol 49, 547-553.

8. Hsia J, Otvos JD, Rossouw JE, et al. (2008) Lipoprotein particle concentrations may explain the absence of coronary protection in the women's health initiative hormone trials. Arterioscler Thromb Vasc Biol 28, 1666-1671.

9. Packard CJ \& Shepherd J (1997) Lipoprotein heterogeneity and apolipoprotein B metabolism. Arterioscler Thromb Vasc Biol 17, 3542-3556.

10. Mora S, Szklo M, Otvos JD, et al. (2007) LDL particle subclasses, LDL particle size, and carotid atherosclerosis in the Multi-Ethnic Study of Atherosclerosis (MESA). Atherosclerosis 192, 211-217.

11. Roche HM \& Gibney MJ (2000) Effect of long-chain n-3 polyunsaturated fatty acids on fasting and postprandial triacylglycerol metabolism. Am J Clin Nutr 71, 232S-237S.

12. Calder PC (2004) n-3 Fatty acids and cardiovascular disease: evidence explained and mechanisms explored. Clin Sci (Lond) 107, 1-11.

13. Psota TL, Gebauer SK \& Kris-Etherton P (2006) Dietary omega-3 fatty acid intake and cardiovascular risk. J Am Coll Cardiol 98, 3I-18I. 
14. Harris WS (1996) n-3 Fatty acids and lipoproteins: comparison of results from human and animal studies. Lipids 31, 243-252.

15. Minihane AM, Khan S, Leigh-Firbank EC, et al. (2000) ApoE polymorphism and fish oil supplementation in subjects with an atherogenic lipoprotein phenotype. Arterioscler Thromb Vasc Biol 20, 1990-1997.

16. Griffin BA (2001) The effect of $n-3$ fatty acids on low density lipoprotein subfractions. Lipids 36, Suppl., S91-S97.

17. Hansen JB, Grimsgaard S, Nilsen H, et al. (1998) Effects of highly purified eicosapentaenoic acid and docosahexaenoic acid on fatty acid absorption, incorporation into serum phospholipids and postprandial triglyceridemia. Lipids 33, 131-138.

18. Mori TA, Burke V, Puddey IB, et al. (2000) Purified eicosapentaenoic and docosahexaenoic acids have differential effects on serum lipids and lipoproteins, LDL particle size, glucose, and insulin in mildly hyperlipidemic men. Am J Clin Nutr 71, 1085-1094.

19. Kelley DS, Siegel D, Vemuri M, et al. (2007) Docosahexaenoic acid supplementation improves fasting and postprandial lipid profiles in hypertriglyceridemic men. Am J Clin Nutr 86, 324-333.

20. Davidson MH (2006) Mechanisms for the hypotriglyceridemic effect of marine omega-3 fatty acids. Am J Cardiol 98, 27I-33I.

21. Lu GP, Windsor SL \& Harris WS (1999) Omega-3 fatty acids alter lipoprotein subfraction distributions and the in vitro conversion of very low density lipoproteins to low density lipoproteins. J Nutr Biochem 10, 151-158.

22. Mostad IL, Bjerve KS, Lydersen S, et al. (2008) Effects of marine $n-3$ fatty acid supplementation on lipoprotein subclasses measured by nuclear magnetic resonance in subjects with type II diabetes. Eur J Clin Nutr 62, 419-429.

23. Calder PC (2007) Immunomodulation by omega-3 fatty acids. Prostaglandins Leukot Essent Fatty Acids 77, 327-335.

24. Bulow J, Simonsen L, Wiggins D, et al. (1999) Co-ordination of hepatic and adipose tissue lipid metabolism after oral glucose. J Lipid Res 40, 2034-2043.

25. Heath RB, Karpe F, Milne RW, et al. (2003) Selective partitioning of dietary fatty acids into the VLDL TG pool in the early postprandial period. J Lipid Res 44, 2065-2072.

26. Burdge GC, Sala-Vila A, West AL, et al. (2007) The effect of altering the $20: 5 n-3$ and $22: 6 n-3$ content of a meal on the postprandial incorporation of $n-3$ polyunsaturated fatty acids into plasma triacylglycerol and non-esterified fatty acids in humans. Prostaglandins Leukot Essent Fatty Acids 77, 59-65.

27. Burdge GC, Powell J \& Calder PC (2006) Lack of effect of meal fatty acid composition on postprandial lipid, glucose and insulin responses in men and women aged 50-65 years consuming their habitual diets. Br J Nutr 96, 489-500.

28. Otvos JD, Jeyarajah EJ \& Cromwell WC (2002) Measurement issues related to lipoprotein heterogeneity. Am J Cardiol 90, 22i-29i.

29. Couture P, Otvos JD, Cupples LA, et al. (2000) Association of the $\mathrm{C}-514 \mathrm{~T}$ polymorphism in the hepatic lipase gene with variations in lipoprotein subclass profiles: The Framingham Offspring Study. Arterioscler Thromb Vasc Biol 20, 815-822.

30. Kraus WE, Houmard JA, Duscha BD, et al. (2002) Effects of the amount and intensity of exercise on plasma lipoproteins. $N$ Engl J Med 347, 1483-1492.

31. Brousseau ME, Schaefer EJ, Wolfe ML, et al. (2004) Effects of an inhibitor of cholesteryl ester transfer protein on HDL cholesterol. N Engl J Med 350, 1505-1515.

32. Bhalodkar NC, Blum S, Rana T, et al. (2004) Comparison of levels of large and small high-density lipoprotein cholesterol in Asian Indian men compared with Caucasian men in the Framingham Offspring Study. Am J Cardiol 94, 1561-1563.

33. Schaefer EJ, McNamara JR, Tayler T, et al. (2002) Effects of atorvastatin on fasting and postprandial lipoprotein subclasses in coronary heart disease patients versus control subjects. Am J Cardiol 90, 689-696.

34. Otvos JD (2002) Measurement of lipoprotein subclass profiles by nuclear magnetic resonance spectroscopy. Clin Lab $\mathbf{4 8}$, $171-180$

35. Heijmans BT, Beekman M, Houwing-Duistermaat JJ, et al. (2006) Lipoprotein particle profiles mark familial and sporadic human longevity. PLoS Med 3, e495.

36. Theobald HE, Goodall AH, Sattar N, et al. (2007) Low-dose docosahexaenoic acid lowers diastolic blood pressure in middle-aged men and women. J Nutr 137, 973-978.

37. The European Medicines Agency CfPMP (2008) Points to consider on adjustment for baseline covariates. http://www.emea. europa.eu/pdfs/human/ewp/286399en.pdf 